\title{
VAC.09 - Improvement of seroneutralization assays throughput and accuracy by the automation of image acquisition and analysis
}

Waleska Dias Schwarcz; ${ }^{1}$ Caio Bidueira Denani ${ }^{1 \star}$; Renan de Oliveira Vieira ${ }^{1}$; Felipe Mendes de Luca ${ }^{1}$; José Henrique Rezende Linhares ${ }^{1}$; Maria Beatriz Junqueira Borges ${ }^{1}$; Sheila Maria Barbosa de Lima ${ }^{1}$.

1Fiocruz/Bio-Manguinhos.

Introduction: Quantification neutralizing antibodies methods are crucial in the development and evaluation of new antiviral vaccines effectiveness and the assay most commonly used is the PlaqueReduction Neutralization Test (PRNT). Although the PRNT is the gold standard tool, it is timeconsuming, not amenable to a large number of samples and difficult to adapt to a high throughput routine. Furthermore, the assay tends to have large variations among different analysts due to plaque recognition and manual counting errors. Therefore, providing a faster and more accurate counting for the neutralizing antibodies would minimize these issues. In this context, innovations such as the automation and associated softwares at critical steps can increase the throughput, reduce the rework and generate more accurate results. Companies specialized in microscopy, robotics and information technology developed a robust system, ScanLab. It consists of a robotic arm coupled to a microscope and a high resolution camera. Scanlab is able to standardize PRNT methods within and between laboratories, reducing the subjectivity and improving the speed and consistency of this test, which could aid the public health response to emerging viral diseases.

Objective: The overall objective is to use ScanLab to implement automation in the image acquisition and quantification steps from Zika microPRN assay. It is aimed to obtain more efficient process and reliable results. The specific objectives are setting the best parameters for image acquisition; developing and validating the automated quantification method using clinical samples.

Methodology: The Zika microPRN assay is performed on microplates, and a plate picture is taken for virus identification, quantification and further virus antibody calculate in samples. Scanlab is used to obtained images and to identify morphological patterns and set the best ranges for its circularity, size, color intensity and other attributes; thus allowing the standardize automated assay. The Scanlab consists of an inverted optical microscope coupled with motorized stage, a high-resolution CCD camera, microplates stackers and a robotic arm. Additionally, softwares controls the image acquisition and virus quantification process. To validate the automated method, its results must be compared to the manual method and there may be maximum of $5 \%$ in R-square coefficient.

Results: The automation process using the Scanlab has already been successfully implemented for Dengue and Yellow Fever microneutralization tests and demonstrated a satisfactory correlation from manual count. It generated a 3 -fold increase in results generation (450/month to around $1200 /$ month) and helped reducing the assay retest rate from $50 \%$ to around $15 \%$. These good preliminary results, and the experience acquired, lead us to believe in transposing successfully the automation process for the Zika microPRN assay.

Conclusion: Scanlab is an automated high-resolution imaging platform engineered to meet the quality requirements for image-based testing. It saves time using robotics and microscopy automation and provides more accurate and reliable results.

Keywords: Automation; Seroneutralization assay; Virus 